\title{
Motivos que dan sentido a aprender en la escuela: la mirada de profesores y estudiantes*
}

\author{
Jorge Valenzuela \\ Carla Muñoz \\ Universidad Católica del Maule. Chile. \\ jvalenzuela@ucm.cl \\ cmunozv@ucm.cl \\ Jorge Miranda-Ossandón \\ Universidad Católica de Temuco. Chile. \\ jmiranda@uct.cl
}

Recibido: 6/11/2019

Aceptado: $19 / 5 / 2020$

Publicado: 29/1/2021

\section{Resumen}

El presente análisis cuantitativo caracteriza y contrasta las lógicas bajo las cuales se otorga sentido a aprender en el contexto escolar chileno. Para realizarlo se consideró una muestra de 181 alumnos de ambos sexos de tercer año medio (K11), de nivel socioeconómico bajo y alto, de la ciudad de Santiago de Chile. Adicionalmente se consideró, para fines comparativos, una muestra de 207 profesores en formación y 297 en ejercicio. Se contrastaron los perfiles de estudiantes de nivel socioeconómico alto y bajo, y de ellos respecto a profesores. Los resultados muestran diferencias en los dos casos. Especialmente relevante es que estas se acentúan cuando se trata de alumnos de nivel socioeconómico bajo. Finalmente, se discuten estos hallazgos y las implicancias de las valoraciones disímiles entre profesores y estudiantes respecto a los motivos para aprender en la escuela.

Palabras clave: motivación; aprendizaje; sistema escolar; profesores; nivel socioeconómico

Resum. Motius que donen sentit a aprendre a l'escola: la mirada de professors $i$ estudiants

La present anàlisi quantitativa caracteritza i contrasta les lògiques en base a les quals s'atorga sentit a aprendre en el context escolar xilè. Per realitzar-la es va considerar una mostra de 181 alumnes de tots dos sexes de tercer any mitjà (k-11), de nivell socioeconòmic baix i alt, de la ciutat de Santiago de Xile. A més, es va dur a terme una comparació d'una mostra de 207 professors en formació i 297 en exercici. Es van contrastar perfils d'estudiants de nivell socioeconòmic alt i baix, i d'ells respecte a professors. Els resultats mostren diferències en tots dos casos. Especialment rellevant és que les diferències entre professors $i$ estudiants s'accentuen quan es tracta d'alumnes de nivell socioeconòmic baix. Finalment, es discuteixen aquestes troballes i les implicacions de les valoracions dissemblants entre professors i estudiants sobre els motius per aprendre a l'escola.

Paraules clau: motivació; aprenentatge; sistema escolar; professors; nivell socioeconòmic

* Esta investigación ha sido financiada por la ANID-Chile a través de los proyectos Fondecyt Regular 1150533 y 1181159. 
Abstract. Motives that give meaning to learning in school: The view of teachers and students

This study characterizes and contrasts the logic under which a sense of learning is assigned in the Chilean school context. A comparison is made of the profiles of students of high and low socio-economic status, as well as between the students and the teachers. For this purpose, we studied a sample of 181 third-year high school students of both sexes (K-11) and of low and high socio-economic status from the city of Santiago de Chile. Additionally, a sample of 207 pre-service teachers and 297 in-service teachers was considered for comparative purposes. The results show differences among the students and between the students and the teachers. Especially relevant is that the differences between teachers and students are accentuated in relation to students of low socio-economic status. Finally, these findings and the implications of the dissimilar evaluations between teachers and students regarding the reasons for learning in the school are discussed.

Keywords: motivation; learning; school system; teachers; socio-economic status

\author{
Sumario \\ 1. Introducción 4. Discusión \\ 2. Método Referencias bibliográficas
}

3. Resultados

\title{
1. Introducción
}

En el aula [...] manipulamos principalmente el sentido: si se construye, al menos temporalmente y parcialmente, el aprendizaje se hace posible; si no, ¡perpetuamos la comedia del saber! (Perrenoud, 1993)

La motivación como constructo psicológico ha sido abordada desde diferentes perspectivas teóricas. Entre ellas, las más clásicas son la teoría de metas (Johnson y Sinatra, 2014; Lee y Turner, 2016), la teoría de la autodeterminación (Ryan y Deci, 2017), la teoría de la autoeficacia (Borzone, 2017; Kok et al., 2014; Stajkovic et al., 2018; Vancouver, Alicke y Halper, 2018) y la teoría de las atribuciones causales (Graham y Taylor, 2016; Weiner, 2008, 2014). Estos distintos enfoques tienen en común una fuerte capacidad para la explicación de diferencias individuales (Elliot, Dweck y Yeager, 2018; Hattie, 2009). No obstante, dichas aproximaciones no serían tan útiles para explicar las diferencias de aprendizaje por nivel socioeconómico (en adelante, NSE). En este sentido, datos recogidos en estudiantes chilenos (Valenzuela, 2006) mostraban que el factor motivacional, asumido desde las perspectivas de la motivación del logro y de la autoeficacia, no darían cuenta de las diferencias que existen en el aprendizaje escolar entre los alumnos pobres y sus pares de situación acomodada. El desafío que plantea la cuestión anterior no trata de explicar el fenómeno más allá de una interpretación basada en la 
intensidad de la motivación de los alumnos (cuán motivados o desmotivados están), sino más bien desde los tipos de motivos que orientan su acción.

La teoría clásica de metas ha subrayado, de manera complementaria a la de la intensidad de la motivación, que ha de tenerse en consideración la orientación de los motivos (Elliot et al., 2018). En esta línea, se ha constatado que la orientación hacia el logro puede darse a través de dos tipos de objetivos o metas: las metas de aprendizaje (mastery, learning o task goals) y las de rendimiento (performance, ego o ability goals), esquema que se ve complementado por otro eje: aproximación y evitación (Eder, Elliot y Harmon-Jones, 2013; Méndez-Giménez et al., 2018). Los resultados en esta línea de trabajo han mostrado que efectivamente la orientación hacia el resultado tiene un efecto menor sobre el desempeño que la orientación hacia el aprendizaje (para una síntesis sobre dicha cuestión, véase Robbins et al., 2004; Urdan y Schoenfelder, 2006). Sin embargo, una de las dificultades que plantea esta perspectiva es que el esquema de análisis se sitúa en el plano de la tarea, es decir, responde a la pregunta por los motivos que impulsan u orientan la conducta de los alumnos para desarrollar tareas específicas, como pueden ser aquellas solicitadas por el profesor en el aula, y no necesariamente por los motivos que activan los recursos cognitivos para aprender en el marco de la actividad escolar.

En virtud de lo anterior, y entendiendo que algo adquiere sentido para el sujeto cuando "tiende a, se orienta a, sirve para, significa algo" (Marquínez, 1980 , p. 142), se propone un análisis específico de la orientación al aprendizaje, distinguiendo grupos de motivos que dan sentido al aprendizaje escolar (en adelante, motivos SAE). Dicho de otro modo, los motivos que dan sentido a aprender los contenidos propuestos por la institución escolar es posible distinguirlos y agruparlos, además de generar un perfil a partir de su valorización que permita tener una mirada complementaria a la motivación escolar (Valenzuela, 2007, 2009). Esta perspectiva se sustenta en la idea de que los alumnos conferirían sentido al aprendizaje (Delannoy, 2005) a partir de las representaciones sobre la institución escolar y de los aprendizajes que esta propone (Hernández, 2010; Neut, Rivera y Miño, 2019; Tiramonti y Minteguiaga, 2004).

Para efectos del estudio, entenderemos por representaciones aquellos conjuntos sociocognitivos o conjuntos organizados de cogniciones (Caravaca Morera et al., 2015; Castorina, 2016; Sammut et al., 2015), referidos, en este caso, al ámbito de la institución escolar y a su eje articulador por excelencia: el aprendizaje académico. Dichas representaciones son adquiridas a través de la experiencia académica y familiar, que si bien son modificables, pareciera que tienden a consolidarse a través del proceso escolar (Abraham, 2004).

\subsection{Tipos de motivos que dan sentido al aprendizaje escolar}

Los motivos que dan sentido al aprendizaje escolar (Coll, 2014; Delannoy, 2005; Develay, 2015; Guzmán Gómez y Saucedo Ramos, 2015; Silva-Peña et al., 2013) y por los cuales un alumno querría adquirir los contenidos plan- 
teados por la institución docente, pueden ser agrupados, según la propuesta de Valenzuela (2009), en cinco tipos orientados a la responsabilidad social, el desarrollo personal, el ascenso social, la sobrevivencia y, finalmente, el mal menor. La identificación de estos motivos permite generar un perfil alternativo de la motivación escolar.

El primero de estos grupos de motivos que dan sentido al aprendizaje escolar ha sido el de responsabilidad social. En este caso, aprender en la escuela se basa en la intención de poner dichos conocimientos al servicio de los demás. Bajo esta lógica el alumno quiere aprender y formarse para contribuir al avance de su comunidad, su región o su país.

El segundo grupo de motivos se inscriben bajo la lógica del desarrollo personal. En este caso, aprender en la escuela tiene sentido en la medida en que la adquisición de conocimientos tiene que ver con la realización personal, con ser mejor ser humano y con aprovechar las propias capacidades.

Un tercer grupo de motivos tiene que ver con el ascenso social y da cuenta de la valoración de aprender en la escuela como medio para mejorar la situación socioeconómica en un futuro próximo. No se trata, evidentemente, de una utilidad inmediata de dichos conocimientos, sino más bien de la percepción de que el conjunto de su formación lo habilitará de la mejor manera posible para conseguir su propósito.

El cuarto de estos sentidos atribuidos a aprender en la escuela está relacionado con la sobrevivencia. Este grupo de motivos se articulan en torno a la visión de que la institución escolar está para darle las herramientas (mínimas) para que pueda integrarse en la sociedad a través del trabajo.

Finalmente, el último grupo de motivos, que se categoriza como mal menor, está vinculado a la concepción de que aprender en la escuela tiene valor, fundamentalmente, como medio para lograr otros fines, normalmente de carácter contingente y cuyo agente es otra persona. En este sentido, lo típico sería que aprender en la escuela sirviera para lograr permisos, premios, evitar castigos o las molestias que se generan por la presión de los padres, etc.

\subsection{La hipótesis de la consonancia de motivos}

Cuando un profesor, en tanto mediador de los aprendizajes, propone ciertos contenidos a los alumnos actúa necesariamente bajo una determinada lógica. Ello no solo se verifica a través del discurso del docente, sino también a través de su comportamiento (Urhahne, 2015). Para el profesor, la apropiación por parte de los estudiantes de los conocimientos de su especialidad tiene evidentemente sentido para él. Pero ¿es el mismo sentido que tiene para los alumnos?, ¿coincide la valoración del profesor con aquella que hacen sus alumnos? Así, en la medida en que coincidan los motivos para aprender (alumnos) y los motivos por los cuales se debería querer aprender (profesores) será posible potenciar el valor de adquirir nuevos conocimientos. En caso contrario, incluso se podría invisibilizar, deslegitimar o restar potencia a los motivos por los cuales un estudiante activa sus recursos cognitivos. 
Constatamos que muchas veces, en el caso de los docentes, los contenidos son intencionados bajo la lógica de motivos ligados al desarrollo personal, mientras que, para el alumno, aprenderlos cobra sentido en la medida en que se aprecia una utilidad práctica por parte del estudiante (Jiménez y Gutiérrez, 2017). ¿Cuántas veces al profesor no le han planteado la pregunta: "¿y para qué sirve aprender esto?»?

Claro que, si la respuesta a la pregunta sobre la utilidad del aprendizaje de los contenidos curriculares está modulada bajo una lógica diferente a la del alumno, habrá un desencuentro que lamentablemente subvalorará la intencionalidad del aprendizaje asignada por aquel. Un ejemplo de ello es cuando el profesor estima que dicho contenido es importante aprenderlo, puesto que contribuye al desarrollo personal del estudiante, mientras que este, por su parte, activa sus recursos para aprender bajo una lógica de utilidad diferente, por ejemplo: que dicho contenido «sirve» o contribuye (con las mediaciones del caso) a ascender socialmente, o al menos es útil para encontrar empleo (SAE sobrevivencia). Este tipo de situaciones genera desencuentros entre las intencionalidades del alumno y las del profesor, pues este último transmite sutil e implícitamente al primero que sus razones para aprender no son válidas.

\subsection{El presente estudio}

Teniendo en consideración este marco, además de describir el comportamiento de los participantes de la muestra en estas variables, nos hemos propuesto testear dos hipótesis. En primer lugar, que los perfiles motivacionales que surgen de la valoración de estos cinco tipos de motivos que dan sentido al aprendizaje escolar son significativamente distintos entre grupos de alumnos de nivel socioeconómico bajo y alumnos de nivel socioeconómico alto, y en segundo lugar, que la valoración de los motivos que realizan los profesores y los estudiantes es distinta.

\section{Método}

\subsection{Participantes}

Para realizar este análisis se consideró una muestra de 181 alumnos de ambos sexos de tercer año medio $(16,4$ años; $\mathrm{SD}=0,63)$, de nivel socioeconómico bajo $(n=78$; mujeres $=48,7 \%)$ y de nivel socioeconómico alto $(n=103$; mujeres $=54,4 \%)$ de la ciudad de Santiago de Chile. Estos jóvenes, respectivamente, corresponden a estudiantes de un establecimiento educacional municipal de una comuna pobre de la ciudad y considerado, para efectos del Sistema Nacional de Medición de Calidad de la Educación (SIMCE), con la categoría socioeconómica $\mathrm{B}$, mientras que los jóvenes de nivel socioeconómico alto pertenecen a dos establecimientos particulares de la zona alta de la ciudad y cuya categorización SIMCE es de E (Darville, Díaz y Melis, 2003).

Adicionalmente se consideró, para fines comparativos, una muestra independiente de 207 profesores en formación (mujeres = 89,9\%, 21,55 años; 
$\mathrm{SD}=2,98)$ y 297 profesores en ejercicio (mujeres $=74,8 \% ; 41,57$ años; $\mathrm{SD}=11,90)$. La muestra abarcó docentes de diferentes niveles y especialidades (todas las áreas escolares). Para efectos prácticos se los consideró como una sola muestra, dado que no presentaban ninguna diferencia significativa entre los que estaban en formación y en ejercicio respecto a los motivos SAE $(p>0,05)$. De igual modo, dentro de esta muestra tampoco se observaron diferencias en los motivos SAE al compararlos según el sexo, la edad, los años de experiencia, el tipo de universidad de origen (selectiva o no selectiva; regional o de la capital) o las áreas disciplinarias. Así, pese a la diversidad de profesores, respecto a las variables evaluadas se comportan de manera similar, por lo que constituyen un grupo homogéneo.

\subsection{Instrumentos}

Para medir el grado de adhesión a los distintos motivos que dan sentido al aprendizaje escolar (motivos SAE) se utilizó la versión actualizada de la escala SAE-Alumno (Valenzuela, 2009). Este instrumento consta de 21 ítems tipo Likert (con escala de 1 a 6 ) y mide el grado de adhesión a cinco tipos de motivos para aprender en la escuela: de responsabilidad social, de desarrollo personal, de ascenso social, de sobrevivencia y, finalmente, de mal menor. La escala presenta una estructura factorial coincidente con la propuesta teórica, CFA: $\chi 2\left({ }_{176}\right)=309,03, p<0,001 ; \chi 2 / \mathrm{gl}=1,75$; NFI = 0,95; CFI = 0,98; RMSEA $=0,040$ [0,032-0,047]; SRMR = 0,04, y buenos niveles de discriminación.

La escala muestra índices de confiabilidad, medidos como confiabilidad compuesta $\mathrm{CR}=0,826 ; 0,702 ; 0,786 ; 0,776$ y 0,689 , y alfas de Cronbach, $\alpha=0,847 ; 0,757 ; 0,851 ; 0,837$ y 0,647 , respectivamente, para cada una de las dimensiones. Cabe consignar que el nivel de confiabilidad es equivalente en ambas muestras, salvo para la escala SAE-A (motivos de responsabilidad social), donde se observan diferencias significativas entre los alfas (Hakstian y Whalen, 1976), entre los estudiantes de nivel socioeconómico bajo $(\alpha=0,777)$ y de nivel socioeconómico alto $(\alpha=0,895), X^{2}$ de 9,$235 ; p=0,002$. Con todo, ambos valores se encuentran por encima de los estándares habituales para este indicador (Field, 2013; Quero Virla, 2010).

Ejemplos de ítems de este instrumento:

Mi principal razón para aprender en el colegio es...

- Porque me gusta aprender cosas nuevas (desarrollo personal).

- Porque me ayuda a conseguir permisos (mal menor).

- Porque me ayudará a tener más opciones de trabajo (sobrevivencia).

Para el caso de la muestra de profesores se utilizó un instrumento equivalente al utilizado para los estudiantes, en el cual solo se cambia «mi principal razón...» por «la principal razón de mis alumnos debería ser...».

De manera complementaria, el instrumento de los profesores consideró una pregunta adicional: «mis estudiantes comparten la valoración que acabo 
de hacer», que buscó establecer la percepción que los docentes tienen del grado de sintonía entre sus valoraciones de los motivos para aprender y las de los estudiantes.

\subsection{Procedimientos}

La administración de este instrumento estuvo a cargo del investigador responsable y se realizó colectivamente durante el horario de clases, sin la presencia del profesor de la asignatura. A los alumnos se les aseguró confidencialidad y la duración de dichas aplicaciones no excedió los 20 minutos.

Tras la invitación a colaborar con esta investigación, se entregó un facsímil del cuestionario a cada estudiante y se leyó y se explicó el protocolo de presentación del instrumento insistiendo especialmente en el carácter confidencial de los resultados individuales y que lo importante era lo que de verdad opinaba cada uno sobre lo que se preguntaba.

A medida que los alumnos iban terminando, se les instaba a comprobar que todas las preguntas estuviesen contestadas, a continuación podían entregar el instrumento. Este procedimiento se realizó de manera similar en todos los cursos evaluados.

En el caso de los profesores, la toma de datos se realizó también de manera colectiva y resguardando los aspectos éticos a través de un consentimiento informado. Su participación fue retribuida por medio del sorteo de una tableta electrónica entre los participantes.

\section{Resultados}

\subsection{Diferencias en el sentido del aprendizaje escolar según el nivel socioeconómico}

Las medias en cada uno de los distintos tipos de motivos SAE se organizan de manera distinta en los estudiantes de NSE alto y bajo (ver figura 1). Lo interesante de estos resultados es que los de NSE alto puntúen los motivos de sobrevivencia a la par que los de desarrollo personal.

Figura 1. Orden jerárquico de tipos de motivos SAE por NSE

\begin{tabular}{|c|c|c|c|}
\hline Orden & NSE bajo & Orden & NSE alto \\
\hline 1 & Sobrevivencia (SAE-D) & $1^{*}$ & Desarrollo personal (SAE-B) \\
\hline 2 & Desarrollo personal (SAE-B) & $1^{*}$ & Sobrevivencia (SAE-D) \\
\hline 3 & Ascenso social (SAE-C) & 3 & Responsabilidad social (SAE-A) \\
\hline 4 & Responsabilidad social (SAE-A) & 4 & Ascenso social (SAE-C) \\
\hline 5 & Mal menor (SAE-E) & 5 & Mal menor (SAE-E) \\
\hline
\end{tabular}

Fuente: elaboración propia. 


\subsection{Motivos que dan sentido al aprendizaje por NSE}

Una segunda aproximación a las diferencias entre estos dos grupos, y que nos permite matizar el análisis anterior, es considerar las medias de los motivos SAE y la varianza asociada a ellos.

Una primera constatación es que los estudiantes de NSE bajo obtienen siempre puntuaciones más altas que sus pares de NSE alto. Por ejemplo, el motivo SAE más valorado por el NSE alto obtiene una puntuación inferior al de las 2 primeras valoraciones del NSE alto bajo, y por otra parte su motivo SAE menos valorado es 0,248 puntos inferior al del grupo de NSE alto bajo (ver tabla 1).

Esta diferencia nos hizo preguntar por un eventual sesgo en la confiabilidad de la medida entre estos dos grupos. Tras comparar los índices de consistencia interna de las cinco escalas en ambos grupos, solo se observó una diferencia significativa en el motivo SAE-A (responsabilidad social), en donde el grupo NSE bajo tiene una alfa de Cronbach significativamente menor a la del grupo alto $\chi^{2}=9,59 ; p=0,002$. No obstante, estos valores $(\alpha=0,777 \mathrm{y}$ $\alpha=0,891$, respectivamente) son lo suficientemente altos como para considerarlos adecuados y para descartar un sesgo sobre la medida.

Respecto a la dispersión de los datos, es interesante hacer notar que los motivos SAE que encabezan la jerarquía tienden a mostrar una menor dispersión que los sentidos ubicados más abajo en la escala. De hecho, se observa una alta correlación entre el orden de los diferentes motivos SAE y las dispersiones correspondientes $(r=0,734 ; p<0,015)$. Esto puede apoyar la idea de que los sentidos mejor evaluados son, a la vez, sentidos consolidados y compartidos grupalmente, y eventualmente, los más estables en el tiempo.

Tabla 1. Medias y dispersión para motivos SAE por nivel socioeconómico

\begin{tabular}{llcc}
\hline NSE bajo & NSE alto & Media & SD \\
\hline Sobrevivencia & & 5,40 & 0,72 \\
\hline Desarrollo personal & & 5,08 & 0,89 \\
& Desarrollo personal & 4,88 & 0,78 \\
\hline Ascenso social & & 4,81 & 0,89 \\
\hline & Sobrevivencia & 4,67 & 1,07 \\
\hline Responsabilidad social & & 3,67 & 1,10 \\
\hline & Responsabilidad social & 3,59 & 1,21 \\
\hline Mal menor & Ascenso social & 2,78 & 1,08 \\
\hline & & 2,43 & 1,16 \\
\hline
\end{tabular}

Fuente: elaboración propia. 


\subsection{Diferencias de medias para cada tipo de motivo $S A E$}

Como se observa en la tabla 2, las únicas diferencias significativas $(p<0,001)$ entre los estudiantes de niveles socioeconómicos bajo y alto las encontramos en los motivos SAE tipo C (ascenso social) y tipo D (sobrevivencia). Son precisamente los primeros quienes valoran significativamente más que sus pares de NSE alto los motivos de ascenso social $\left(\eta^{2}=0,498\right)$ y de sobrevivencia $\left(\eta^{2}=0,137\right)$.

Tabla 2. Comparación de motivos SAE por NSE

\begin{tabular}{lccccc}
\hline & Sig. Levene & $\mathbf{F}$ & Sig. & $\eta^{2}$ & Potencia \\
\hline Responsabilidad social (SAE-A) & 0,605 & 0,391 & 0,533 & 0,002 & 0,095 \\
\hline Desarrollo personal (SAE-B) & 0,347 & 1,897 & 0,170 & 0,011 & 0,278 \\
\hline Ascenso social (SAE-C) & 0,073 & 17,912 & 0,001 & 0,498 & 1,000 \\
Sobrevivencia (SAE-D) & 0,001 & 27,193 & 0,001 & 0,137 & 0,999 \\
\hline Mal menor (SAE-E) & 0,042 & 1,936 & 0,166 & 0,011 & 0,283 \\
\hline
\end{tabular}

Fuente: elaboración propia.

\subsection{Diferencias en la valoración de motivos SAE entre los estudiantes $y$ los profesores}

Una segunda etapa de este estudio tenía que ver con contrastar los resultados de ambos grupos de estudiantes (NSE alto y bajo) con la valoración de cada uno de estos cinco tipos de motivos para aprender en la escuela, pero esta vez estimada por parte del profesorado (ver figura 2). Cabe consignar que para

Figura 2. Medias por tipos de motivos SAE para estudiantes de NSE bajo, alto y profesores

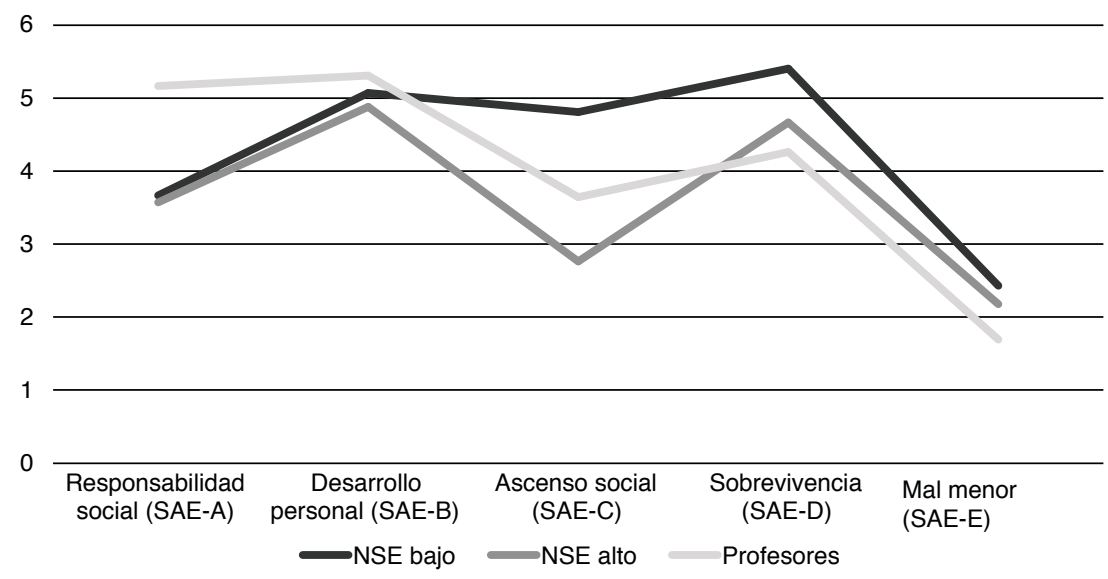

Fuente: elaboración propia. 
este análisis reunimos en un mismo grupo a profesores en formación y en ejercicio, ya que entre ellos no se evidenciaron diferencias significativas respecto a los motivos SAE. Tampoco se evidenciaron diferencias significativas en estas escalas por sexo, región, tipo de universidad de origen o especialidad disciplinaria de los docentes.

Los resultados muestran diferencias significativas $(p<0,05)$ entre los profesores y entre ambos grupos de estudiantes en todos los tipos de SAE, con excepción de SAE-B (desarrollo personal), donde los profesores se diferencian solo de los estudiantes de NSE bajo (ver los subconjuntos homogéneos en la tabla 3). Así, en el caso de los motivos de responsabilidad social esta diferencia se refiere a ambos grupos de estudiantes, tal y como ocurre de manera inversa en los motivos de sobrevivencia y mal menor. En el caso de los motivos de desarrollo personal, la diferencia que se observa es entre profesores y estudiantes de NSE alto. Por su parte, los profesores valoran significativamente menos los motivos de ascenso social que los estudiantes de NSE bajo, pero de modo relevante más que los estudiantes de NSE alto.

Tabla 3. Medias y subconjuntos homogéneos por grupos

\begin{tabular}{lccc}
\hline & NSE bajo & NSE alto & Profesores \\
\hline Responsabilidad social (SAE-A) & $3,67^{\mathrm{a}}$ & $3,58^{\mathrm{a}}$ & $5,17^{\mathrm{b}}$ \\
Desarrollo personal (SAE-B) & $5,08^{\mathrm{ab}}$ & $4,88^{\mathrm{a}}$ & $5,32^{\mathrm{b}}$ \\
\hline Ascenso social (SAE-C) & $4,81^{\mathrm{c}}$ & $2,77^{\mathrm{a}}$ & $3,64^{\mathrm{b}}$ \\
\hline Sobrevivencia (SAE-D) & $5,40^{\mathrm{c}}$ & $4,67^{\mathrm{b}}$ & $4,26^{\mathrm{a}}$ \\
\hline Mal menor (SAE-E) & $2,43^{\mathrm{b}}$ & $2,18^{\mathrm{b}}$ & $1,69^{\mathrm{a}}$ \\
\hline
\end{tabular}

$a, b$ y $c$ : subconjuntos homogéneos $p>0,05$.

Fuente: elaboración propia.

Finalmente, es importante consignar que al final del instrumento para profesores se agregó un ítem tipo Likert de 6 puntos, en el cual se les consultaba: «mis estudiantes comparten la valoración que acabo de hacer». Los resultados muestran que los profesores creen mayoritariamente que sus estudiantes comparten su valoración de motivos para aprender $(M=5,00, S D=1,12)$, esto se traduce en que solo un 10,6\% de los profesores en ejercicio y un 12,1\% de los futuros profesores creen que los estudiantes valoran de manera distinta a ellos los diversos motivos para aprender en la escuela (puntuaciones en la mitad inferior de la escala).

\section{Discusión}

El presente estudio se centró en testear dos hipótesis principales. La primera de ellas señala que los perfiles motivacionales surgidos de la valoración de estos cinco tipos de motivos que dan sentido al aprendizaje escolar son significativamente distintos en grupos de alumnos de NSE bajo y alumnos de NSE alto $y$, en segundo lugar, que esta valoración de los motivos SAE era, a su vez, 
distinta de la que asignan los profesores a cada uno de los tipos de motivos. Los resultados nos ayudan a confirmar parcialmente ambas hipótesis, pero además han proporcionado resultados interesantes a nivel descriptivo acerca de cómo se valoran los distintos motivos que dan sentido a aprender en el contexto escolar.

Así, se constata que el grupo de alumnos de nivel socioeconómico bajo tiende a asignar sentido al aprendizaje escolar valorando significativamente más la sobrevivencia o la integración a la sociedad en función de la perspectiva laboral y la mejora de su situación socioeconómica. Es decir, estos estudiantes activarían sus recursos cognitivos para aprender los contenidos escolares seleccionando lo «que me va a servir», «lo útil», muy posiblemente influidos por su realidad social, que impone la satisfacción de necesidades más básicas o urgentes. Lo anterior puede también estar potenciado, adicionalmente, por el hecho de que estos estudiantes están próximos a terminar su etapa escolar y esto puede añadir una cuota adicional de incertidumbre a sus consideraciones.

Respecto de la segunda hipótesis, se evidenciaron diferencias significativas entre la valoración de los profesores y la de los alumnos. Estas diferencias de valoración de los tipos de motivos para aprender evidencian una distancia mayor entre los profesores y los estudiantes de NSE bajo, que respecto a los estudiantes de NSE más acomodado. Esta podría ser una pista para hipotetizar una mejor sintonía entre los profesores y los estudiantes con más oportunidades, sintonía que se podría traducir en un refuerzo de los motivos para interiorizar los contenidos del currículum. Por el contrario, la distancia mayor con los estudiantes más desfavorecidos podría, de una manera implícita y no deseada, fragilizar los motivos que los estudiantes de NSE bajo tienen para aprender, dado que los profesores actúan como un otro significativo a la hora de validar los motivos para querer adquirir conocimientos en la escuela.

Otra constatación importante respecto de este objetivo es que ni los profesores en ejercicio ni aquellos que se encuentran en formación asumen que sus estudiantes (y especialmente los de NSE bajo) pudieran asignar sentido a aprender en la escuela de una manera significativamente diferente a ellos. En este contexto, un primer desafío es tomar conciencia de las lógicas bajo las cuales los alumnos efectivamente asignan sentido a aprender. En esta perspectiva, el hecho de poder conocer cómo valoran los alumnos cada uno de estos tipos de motivos que dan sentido a aprender permitiría tomar decisiones pedagógicas para «sintonizar» o reducir una eventual brecha entre la lógica bajo la cual se enseña respecto a la cual se aprende, y así poder alcanzar aprendizajes más significativos (Esteban-Guitart, 2016) y profundos (Álvarez Cedillo et al., 2019).

En este contexto, a la diferencia en la valoración de los motivos de aprender entre profesores y estudiantes, especialmente respecto a los de NSE bajo, se suma la falta de conciencia de los docentes de que los motivos que ellos valoran no son necesariamente considerados de la misma manera por el alumnado.

Es posible que el valor de lo prescriptivo asociado a la profesión docente impida muchas veces percibir que la aproximación motivacional de los estu- 
diantes respecto al aprendizaje pueda ser distinta a la que ellos consideran válida (la que debe ser).

Evidentemente, no se trata de imponer los «buenos» motivos para aprender en la escuela ni de conformarse con aquellos motivos que tenga el alumno. Tomar conciencia de que abordamos el aprendizaje escolar con lógicas distintas permitiría la posibilidad de 1) no desvalorizar el recurso motivacional con el que el alumno quiere aprender y 2), a partir de la constatación de la diferencia, enriquecer la gama de sentidos ofreciendo de manera complementaria un abanico más amplio de motivos para apropiarse de los contenidos que la escuela le propone.

De esta manera, pareciera que preguntarse por los «intereses» de nuestros alumnos no fuese la cuestión más importante. Lo relevante parecería ser comprender bajo qué lógica movilizarían sus recursos cognitivos y actitudinales para aprender lo que la escuela les propone. Dicho de otro modo: qué motivos dan sentido al aprendizaje (Coll, 2014; Develay, 2015; Falsafi y Coll, 2015; Kaplan, 2013)

Sabemos que los alumnos de nivel socioeconómico bajo tienden a tener un desempeño menor que sus compañeros de clase acomodada (OECD, 2016; Otero, Carranza y Contreras, 2016). Esto no solo podría deberse al capital cultural fruto de las oportunidades que brinda la familia o la escuela (Rosas y Santa Cruz, 2013), sino que podría estar mediado por la falta de sentido (Develay, 2015; Perrenoud, 1993) de los aprendizajes, cuestión que sería oportuno responderse en un futuro estudio.

En síntesis, constatamos que el sentido atribuido a aprender en la escuela no es el mismo para los alumnos de nivel socioeconómico bajo que para los de nivel socioeconómico alto. Esta primera constatación, que a primera vista nos puede parecer evidente, nos lleva a poner de relieve el hecho de que motivar a estos alumnos para aprender será más eficaz si tomamos como punto de partida los motivos actuales por los cuales ellos quieren aprender en la escuela, y desde allí ampliamos su horizonte de sentido dando más y mejores oportunidades de querer aprender. Con todo, esto solo es posible si tomamos conciencia de que nuestros estudiantes, especialmente los más pobres, asignan sentido a aprender de una manera no siempre concordante con el ideal del profesor.

\section{Referencias bibliográficas}

Abraham, M. (2004). La Resignificación de los Procesos de Enseñanza y Aprendizaje de Alumnas y Alumnos de Formación Docente Inicial. Pensamiento Educativo, 35(2), 216-234. Recuperado de <https://tinyurl.com/abraham2014>.

Álvarez Cedillo, J.A.; Álvarez SÁnchez, T.; Sandoval Gómez, R.J. y Aguilar FerNÁNDEZ, M. (2019). La exploración en el desarrollo del aprendizaje profundo. RIDE: Revista Iberoamericana para la Investigación y el Desarrollo Educativo, 9(18), 833-844. $<$ http://dx.doi.org/10.23913/ride.v9i18.474>

Borzone, M.A. (2017). Self-efficacy and academic experiences with university students. Acta Colombiana de Psicología, 20(1), 275-283.

$<$ http://doi.org/10.14718/ACP.2017.20.1.13> 
Caravaca Morera, J.A.; Itayra Padilha, M.; Guerreiro Vieira da Silva, D. y SAPAG, J. (2015). Theoretical and methodological aspects of social representations. Texto \& Contexto-Enfermagem, 24(4), 1157-1165. <http://dx.doi.org/10.1590/0104-0707201500003440014>

Castorina, J.A. (2016). La significación de la teoría de las representaciones sociales para la psicología. Perspectivas en Psicología, 13(1), 1-10. Recuperado de <http:// 200.0.183.216/revista/index.php/pep/article/view/245>.

Coll, C. (2014). El sentido del aprendizaje hoy: Un reto para la innovación educativa. Aula de Innovación Educativa, 232, 12-17. Recuperado de <http://diposit. ub.edu/dspace/bitstream/2445/65763/1/642900.pdf $>$.

Darville, P.; Díaz, P. y Melis, F. (2003). Metodología para agrupar establecimientos por nivel socioeconómico: Prueba Simce 4to Básico, 2002. Santiago: Departamento de Estudios y Estadísticas. Ministerio de Educación.

Delannoy, C. (2005). La Motivation: Désir de savoir et décision d'apprendre. París: CNDP, Hachette Éducation.

Develay, M. (2015). Donner du sens aux savoirs: La didactique, quarante ans après. Educar em Revista, 58, 149-163. <https://dx.doi.org/10.1590/0104-4060.43363>

Eder, A.B.; Elliot, A.J. y Harmon-Jones, E. (2013). Approach and avoidance motivation: Issues and advances. Emotion Review, 5(3), 227-229. <http://doi.org/10.1177/1754073913477990>

Elliot, A.J.; Dweck, C.S. y Yeager, D.S. (2018). Handbook of competence and motivation. 2. ${ }^{\mathrm{a}}$ ed. Nueva York: Guilford Publications.

Esteban-Guitart, M. (2016). Funds of Identity: Connecting Meaningful Learning Experiences in and Out of School. Cambridge: Cambridge University Press.

Falsafi, L. y Coll, C. (2015). Influencia educativa y actos de reconocimiento: La identidad de aprendiz, una herramienta para atribuir sentido al aprendizaje. Papeles de Trabajo sobre Cultura, Educación y Desarrollo Humano, 11(2), 16-19. Recuperado de <https://tinyurl.com/falsafiycoll2015>.

FIELD, A. (2013). Discovering statistics using IBM SPSS statistics. 3. a ed. Londres: Sage.

Graham, S. y TAYlor, A.Z. (2016). Attribution theory and motivation in school. En K. R. Wentzel y D. Miele (eds.). Handbook of Motivation at School (pp. 11-33). Nueva York: Routledge.

Guzmán Gómez, C. y Saucedo Ramos, C.L. (2015). Experiencias, vivencias y sentidos en torno a la escuela y a los estudios: Abordajes desde las perspectivas de alumnos y estudiantes. Revista Mexicana de Investigacion Educativa, 20(67), 1019-1054. Recuperado de <http://www.scielo.org.mx/pdf/rmie/v20n67/v20n67a2.pdf>.

Hakstian, R. y Whalen, T. (1976). A K-Sample Significance Test for Independent Alpha Coefficients. Psychometrika, 41(2), 219-231. <http://doi.org/10.1007/BF02291840>

Hattie, J. (2009). Visible learning: A synthesis of 800+ meta-analyses on achievement. Oxford: Routledge.

Hernández, O.G. (2010). El sentido de la escuela: Análisis de las representaciones sociales de la escuela para un grupo de jóvenes escolarizados de la ciudad de Bogotá. Revista Mexicana de Investigacion Educativa, 15(46), 945-967. Recuperado de $<$ https://tinyurl.com/hernandez2010>.

Jiménez, A. y Gutiérrez, A.S. (2017). Realidades escolares en las clases de matemáticas. Educación Matemática, 29, 109-129.

<http://doi.org/10.24844/em2903.04> 
Johnson, M.L. y Sinatra, G.M. (2014). The Influence of Approach and Avoidance Goals on Conceptual Change. The Journal of Educational Research, 107(4), 312325. <http://doi.org/10.1080/00220671.2013.807492>

Kaplan, A. (2013). El sentido de educar: Identidad, diversidad, diferencia. Novedades Educativas, 271, 12-13.

Kok, G.; Den Boer, D.-J.; De Vries, H.; Gerards, H.J.H. y Mudde, A.N. (2014). Self-Efficacy and Attribution Theory. En R. Schwarzer (ed.). Self-Efficacy: Thought Control of Action. Nueva York: Routledge.

LeE, J. y Turner, J. (2016). The role of pre-service teachers' perceived instrumentality, goal commitment, and motivation in their self-regulation strategies for learning in teacher education courses. Asia-Pacific Journal of Teacher Education, 1-16. <http://doi.org/10.1080/1359866X.2016.1210082>

Marquínez, G. (1980). Metafísica desde Latinoamérica. Bogotá: Universidad Santo Tomás.

Méndez-Giménez, A.; Cecchini-Estrada, J.A.; Méndez-Alonso, D.; PrietoSAborit, J.A. y Fernández-Rio, J. (2018). Efecto de las metas de logro y las estructuras de metas de clase 3 × 2 en la motivación autodeterminada: Un análisis multinivel en educación secundaria. Anales de Psicología / Annals of Psychology, 34(1), 52-62. <http://dx.doi.org/10.6018/analesps.34.1.262131>

Neut, P.; Rivera, P. y Miño, R. (2019). El sentido de la escuela en Chile: La creación de paradigmas antagónicos a partir del discurso de política pública, el discurso académico y la investigación educativa. Estudios Pedagógicos (Valdivia), 45(1), 151-168. <http://dx.doi.org/10.4067/S0718-07052019000100151>

OECD (2016). PISA 2015. Results Excellence and Equity in Education (Volume I). Recuperado de <http://www.oecd.org/education/pisa-2015-results-volume-i9789264266490-en.htm>.

Otero, G.; Carranza, R. y Contreras, D. (2016). Los "efectos del barrio" en el rendimiento educacional de los niños en Chile: Los efectos de la organización local, polarización y desigualdad (pp. 28). Recuperado de <https://tinyurl.com/otero-etal2016>.

Perrenoud, P. (1993). Sens du travail et travail du sens à l'école. Cahiers Pédagogiques, 314-315, 23-27. Recuperado de <http://www.cahiers-pedagogiques.com/Sensdu-travail-et-travail-du-sens-a-l-ecole $>$.

Quero Virla, M. (2010). Confiabilidad y coeficiente Alpha de Cronbach. Telos, 12(2), 248-252. Recuperado de <https://tinyurl.com/quero2010>.

Robbins, S.; Lauver, K.; Le, H.; Davis, D.; Langley, R. y Carlstrom, A. (2004). Do psychosocial and study skill factors predict college outcomes?: A meta-analysis. Psychological Bulletin, 130(2), 261-288. <http://doi.org/10.1037/0033-2909.130.2.261>

Rosas, R. y Santa Cruz, C. (2013). Dime en qué colegio estudiaste y te diré qué CI tienes: Radiografia al desigual acceso al capital cognitivo en Chile. Santiago: Ediciones UC.

Ryan, R.M. y Deci, E.L. (2017). Self-Determination Theory: Basic Psychological Needs in Motivation, Development, and Wellness. Nueva York: Guilford Press.

Sammut, G.; Andreouli, E.; Gaskell, G. y Valsiner, J. (eds.). (2015). The Cambridge handbook of social representations. Cambridge: Cambridge University Press. 
Silva-Peña, I.; Bastidas, K.; Calfuqueo, L.; Díaz, J. y Valenzuela, J. (2013). Sentido de la escuela para niños y niñas mapuche en una zona rural. Polis (Santiago), 12(34), 243-258.

<http://dx.doi.org/10.4067/S0718-65682013000100013>

Stajkovic, A.D.; Bandura, A.; Locke, E.A.; Lee, D. y Sergent, K. (2018). Test of three conceptual models of influence of the big five personality traits and selfefficacy on academic performance: A meta-analytic path-analysis. Personality and Individual Differences, 120, 238-245. <http://doi.org/10.1016/j.paid.2017.08.014>

Tiramonti, G. y Minteguiaga, A. (2004). Una nueva cartografía de sentidos para la escuela. En G. Tiramonti (ed.). La trama de la desigualdad educativa: Mutaciones recientes en la escuela media (pp. 101-118). Buenos Aires: Manantial.

URdAN, T.C. y SchoenfeldDer, E. (2006). Classroom effects on student motivation: Goal structures, social relationships, and competence beliefs. Journal of School Psychology, 44(5), 331-349. $<$ http://doi.org/10.1016/j.jsp.2006.04.003>

URHAHNE, D. (2015). Teacher behavior as a mediator of the relationship between teacher judgment and students' motivation and emotion. Teaching and Teacher Education, 45, 73-82. $<$ http://doi.org/10.1016/j.tate.2014.09.006>

Valenzuela, J. (2006). Enseñanza de Habilidades de Pensamiento y Motivación Escolar: Efectos del Modelo Integrado para el Aprendizaje Profundo (MIAP) sobre la Motivación de Logro, el Sentido del Aprendizaje Escolar y la Autoeficacia. [Tesis doctoral.] Santiago de Chile: Pontificia Universidad Católica de Chile.

- (2007). Más allá de la tarea: Pistas para una redefinición del concepto de Motivación Escolar. Educaçao e Pesquisa, 33(3), 409-426. $<$ http://doi.org/10.1590/S1517-97022007000300002>

- (2009). Características psicométricas de una escala para caracterizar el Sentido del Aprendizaje Escolar. Universitas Psychologica, 8(1), 49-59. Recuperado de <https:// revistas.javeriana.edu.co/index.php/revPsycho/article/view/258>.

Vancouver, J.B.; Alicke, M. y Halper, L.R. (2018). Self-Efficacy. En D. Lance Ferris, R.E. Johnson y C. Sedikides (eds.). The Self at Work: Fundamental Theory and Research (pp. 15-38). Londres: Routledge.

Weiner, B. (2008). Reflections on the History of Attribution Theory and Research. Social Psychology, 39(3), 151-156. $<$ http://doi.org/10.1027/1864-9335.39.3.151>

- (2014). The attribution approach to emotion and motivation: History, hypotheses, home runs, headaches/heartaches. Emotion Review, 6(4), 353-361. $<$ http://doi.org/10.1177/1754073914534502> 